\title{
Adaptive Attenuation Correction in Contrast Echo
}

\author{
G Zwirn $^{1}$, R Beeri ${ }^{2}$, D Gilon ${ }^{2}$, S Akselrod ${ }^{1}$ \\ ${ }^{1}$ Tel Aviv University, Tel Aviv, Israel \\ ${ }^{2}$ Hadassah University Hospital, Ein Kerem, Jerusalem, Israel
}

\begin{abstract}
Contrast echocardiography, as a tool for quantitative evaluation of left ventricular myocardial perfusion, has been the subject of massive research in the last few years. Nevertheless, this method is not in wide clinical use.

Standard procedures derive the regional blood flow from the time-dependency of the echocardiographic videointensity within the myocardium. Thus, the data acquisition protocol tends to be lengthy (at least $5-6$ sec). This is a serious problem, since the imaging plane must not change during the entire data acquisition.

The present study presents a novel method, correcting for the signal attenuation in both the tissue and the contrast agent. It enables direct measurement of the local blood volume within the entire myocardium, based on a single heartbeat. Up until now, our technique has been tested on seven different patients, and shows high correlation between the automatically calculated perfusion levels and the segmental contractility.
\end{abstract}

\section{Introduction}

Quantitative evaluation of left ventricular myocardial perfusion using contrast echocardiography has been the subject of numerous studies for well over a decade (e.g., [1-4]). It has the potential to partially replace SPECT (Single Photon Emission Computerized Tomography) imaging, which is much more expensive and timeconsuming than echocardiography, and to reduce the need for invasive procedures, such as exploratory angiography. Yet, this contrast echo technique is rarely used clinically.

The main obstacle on its way to clinical use is the cumbersome data acquisition protocol. Currently available methods are based on the assumption that the contrast-agent's concentration is linearly correlated to the gray level within the echocardiographic image [3]. However, the ultrasonic signal in attenuated by both the tissue and the contrast agent it traverses, so that the attenuation coefficient for each pixel changes in space and time. Thus, the local gray level cannot be translated directly into the contrast-agent's local concentration, which is a direct perfusion measure. Instead, the local perfusion is estimated according to the temporal variability in the local gray level.

As a result, standard protocols tend to require echocardiographic imaging of a single plane for relatively long periods of time (at least $5-6 \mathrm{sec}$ ). These relatively lengthy periods are required to allow the build-up or the decay time of the contrast agent concentration within the myocardium. During the data acquisition, even small vibrations of the transducer may introduce severe artifacts into the images. Ischemic regions and myocardial infarcts are sometimes quite localized, so that such vibrations may cause each pixel to actually include contributions from different regions, with different levels of perfusion.

The present study proposes a procedure for adaptive attenuation correction in contrast echo. The algorithm calculates the correction coefficients for each small myocardial region in each frame, based on the echocardiographic information for a single heartbeat. Thus, the data acquisition protocol is very user-friendly.

Furthermore, the attenuation correction technique is part of larger project [5], designing a complete procedure for automatically estimating the location of the myocardium in each frame, and producing quantitative information regarding the local perfusion in the entire Left Ventricle (LV) simultaneously.

\section{Methods}

\subsection{The inputs to the procedure}

Basically, the inputs to the attenuation correction procedure are the Integrated Backscatter (IBS) values for each small segment ("mini-segment") of the myocardium, in each frame of the cine-loop. The term IBS usually refers to the local mean gray level in an echocardiographic image. In our case, however, each mini-segment in each frame includes both regions dominated by echoes returned from tissue and regions dominated by echoes returned from the contrast agent. Hence, two IBS values correspond to each mini-segment in each frame - one for the tissue and one for the contrast agent.

As described in [5] (see also [6]), the IBS estimation procedure curve-fits the gray level histogram, for each mini-segment in each frame, to the sum of three Gaussian 
functions (see Fig. 1):

1. Low-intensity tissue pixels (low intensity Gaussian).

2. High-intensity tissue pixels (medium intensity Gaussian).

3. Pixels corresponding to the contrast agent (high intensity Gaussian).

The peak location for the low intensity Gaussian is thus used as the IBS for the tissue, whereas the peak location for the high intensity Gaussian is used as the IBS for the contrast agent.

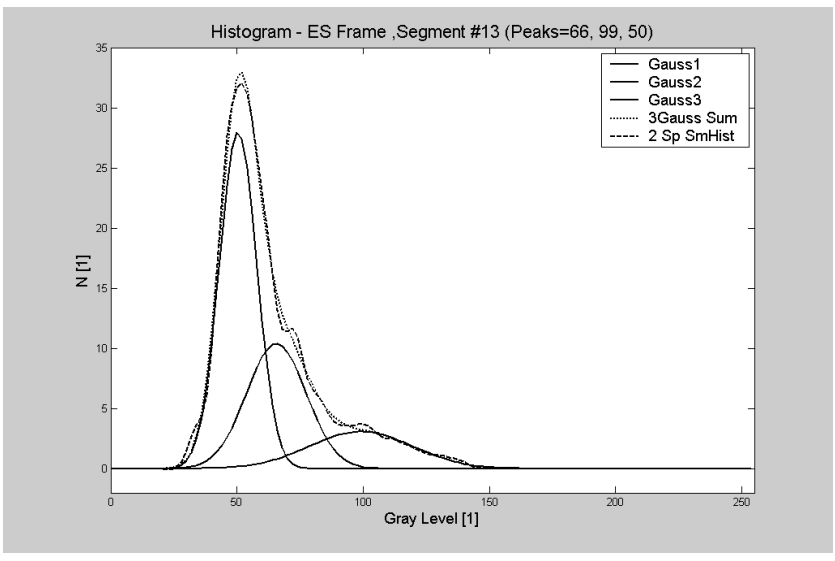

Figure 1. An example for curve-fitting the gray level histogram for a single mini-segment in a single cine-loop. The gray level histogram is shown as a dashed curve, the Gaussian functions are shown as solid curves, and their sum is shown as a dotted curve. Note the close proximity between the actual histogram and the sum of the three Gaussians, supporting the validity of the curve-fit.

\subsection{Adaptive attenuation correction}

Our attenuation correction procedure is based on the assumption, that the temporal average (over an integral number of heartbeats) of the tissue's reflection crosssection (per unit of volume), $\sigma_{t}$, is approximately constant over the myocardium. This postulation is logical, since the entire myocardium is basically of the same tissue type. Consequently, we suggest a coarse (time independent) attenuation correction for each minisegment, based on the median over the tissue's gray level as a function of time, $t_{s, p}$ ( $s$ is the mini-index and $p$ is the frame index). The coarse correction factor, $A_{s}$, which is added to the gray level intensity, is simply the difference between the maximal value of the median, and the value of the median for the specific mini-segment:

$A_{s}=\operatorname{Max}_{s}\left\{\operatorname{Median}_{p}\left(t_{s, p}\right)\right\}-\operatorname{Median}_{p}\left(t_{s, p}\right)$

The same factor can be applied to both the gray level of the contrast agent and of the tissue.

Note that additive correction is used rather than multiplicative correction, since in most imaging platforms, the videointensity is proportional to the logarithm of the magnitude of the returned signal.

As shown hereon (see also [5]), the time independent attenuation correction is sufficient for estimating the local perfusion. Nonetheless, accurate attenuation correction requires temporal dependence.

For that purpose, we hypothesize that the dominant factor in the attenuation correction, in apical two-chamber and apical four-chamber views, is the distance from the transducer, $R(m, n)$. This parameter also determines the local Time Gain Control (TGC) correction, applied in common echocardiographic platforms. Note that another possible parameter might be the distance traversed by the beam through the LV cavity (which is opacified when using our data acquisition protocol), $D(m, n)$.

Thus, after calculating the time independent attenuation correction for each mini-segment $A_{s}$, we update the attenuation correction for each frame according to $R(m, n)$. This is performed by plotting the correction factor $A_{s}$ versus the mean distance from the transducer to the mini-segment, and interpolating the value of $A_{s}$ for the current range of each mini-segment in each frame. For further information - see [7].

\subsection{Quantitative parameter extraction}

A local quantitative measure for the myocardial perfusion, $P_{s}$, can be directly derived from the attenuation corrected IBS. Since the videointensity of the contrast agent is proportional to its local concentration, the difference between the attenuation-corrected gray level of the contrast agent and the attenuation-corrected gray level of the tissue provides insight into the local perfusion. The maximal perfusion level in the cardiac muscle can be used for calibration, assuming it corresponds to $100 \%$ perfusion (this method is also used in SPECT imaging).

An additional parameter, which can be extracted from the attenuation-corrected IBS, is the phase of the cardiac cycle, at which the contrast agent's local gray level has reached its maximal value, $\phi_{\max }^{s}$. The phase is given in percents of the cycle, with respect to End-Systole (ES), determined according to Electrocardiography (ECG). In order to decrease the effects of noise, Smoothing Spline is applied to the data prior to locating the peak.

\subsection{Data acquisition and analysis}

The proposed algorithm has been tested on ten cineloops, belonging to seven different intensive care cardiac unit patients. The cine-loops are in apical four-chamber (seven loops) and apical two-chamber views (three loops). The data has been collected using Vivid-3 and Vivid-7 imaging platforms (GE Healthcare), using low mechanical-index imaging. The contrast agent used is 
Optison Microbubbles (administered at a continuous drip).

For each loop, the local contractility has been clinically evaluated based on echocardiographic imaging. The clinical evaluation has been performed for each displayed cardiac segment separately, using the standard six-segments scheme. Out of the 60 segments (in 10 cineloops), 38 have been defined as normal, 7 as hypokinetic and 15 as akinetic.

In addition, for each cine-loop, the contrast agent's attenuation-corrected gray level has been computed for each mini-segment and frame, and the quantitative parameters, $P_{s}$ and $\phi_{\max }^{s}$, have also been extracted.

Furthermore, we have examined the validity of the assumption, that the dominant factor in the attenuation correction is the distance from the transducer, $R(m, n)$. For that purpose, our coarse correction factor $A_{s}$ has been plotted versus both $R(m, n)$ and $D(m, n)$, for each cineloop. Note that each distance appears twice in both graphs $\left(A_{s}\right.$ versus $R(m, n)$ and $\left.D(m, n)\right)$ - once for the left myocardial wall in the image (e.g., the septum), and once for the right (e.g., the lateral wall). For further corroboration, the Mean Square Error (MSE) between the graphs for the left and the right wall has been calculated.

\section{Results}

\subsection{Procedure validation}

An example for a graph plotting our coarse correction factor $A_{s}$ versus the mean distance traversed by the beam along the LV cavity $D(m, n)$, for each mini-segment (in a specific cine-loop), is shown in Fig. 2. Fig. 3 shows the coarse correction factor $A_{s}$ versus the mean distance from the transducer $R(m, n)$, for each mini-segment (again, in a specific cine-loop).

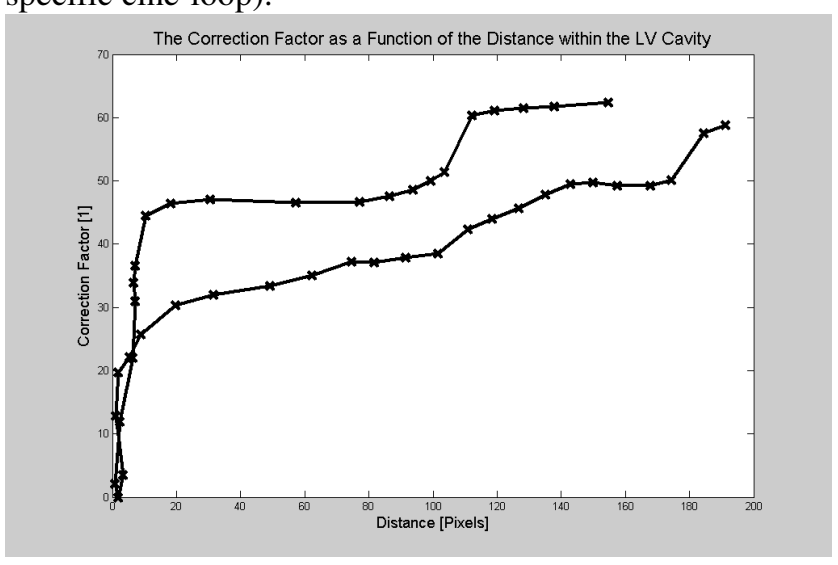

Figure 2. The coarse correction factor versus the mean distance traversed by the beam within the LV cavity, per mini-segment. The two lines represent the two myocardial walls in the echocardiographic image, and they obviously do not match (in most cases, the difference between them is approximately 15 gray levels).

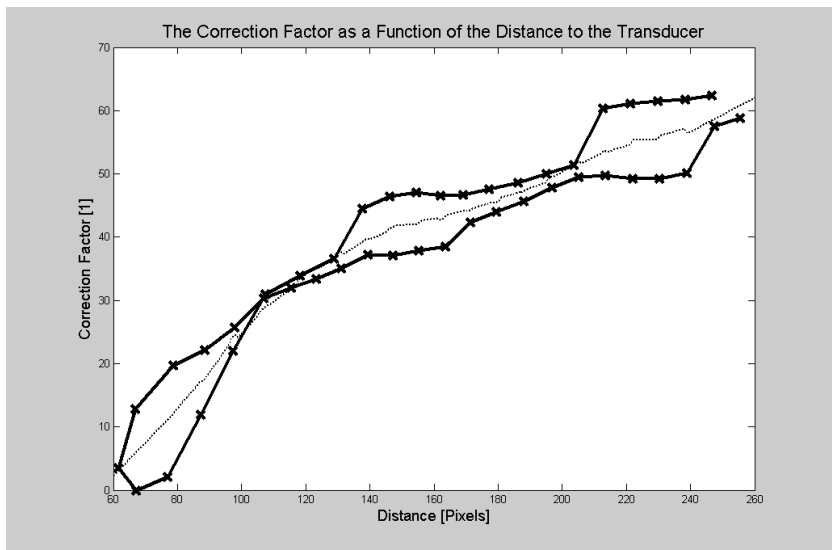

Figure 3. The coarse correction factor versus the mean distance from the transducer, per mini-segment. The two solid lines represent the two myocardial walls in the echocardiographic image, and the difference between them is small. The dotted line shows the final correction factor as a function of the distance from the transducer.

According to six cine-loops, the MSE for graphs of $A_{s}$ versus $R(m, n)$ is $64 \pm 69$, whereas the MSE for the graphs of $A_{s}$ versus $D(m, n)$ is $238 \pm 290$.

\subsection{Data analysis}

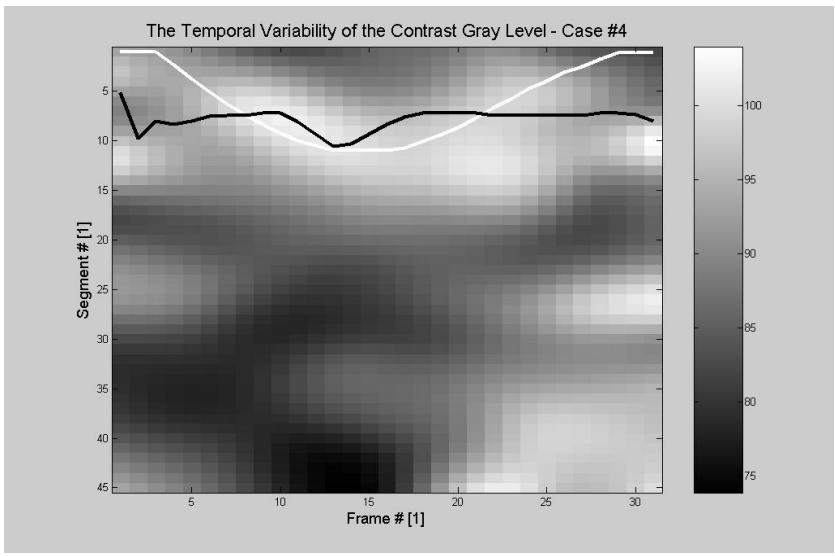

Figure 4. The contrast agent's gray level as a function of the mini-segment index (the Y-axis) and the frame index (the $\mathrm{X}$-axis), after applying attenuation correction. The color-bar on the right defines the actual gray level corresponding to each displayed videointensity. The LV volume, determined according to the estimated boundaries of the LV cavity (using the area-length method), appears on the top in white, whereas the ECG appears on the top in black. In this case, all the segments have been diagnosed as normal. 
An example for the attenuation corrected IBS, as calculated for the entire myocardium in a specific cineloop, is shown in Fig. 4.

Table 1 compares the results of applying a constant threshold $(40 \%)$ to the calculated mean perfusion measure, $P_{s}$, to the clinical evaluation of segmental contractility (for all segments in all the cine-loops).

Table 1. Comparison between the clinical evaluation of contractility, based on echocardiography, and the calculated perfusion measure $P_{s}$.

\begin{tabular}{ccc}
\hline & $\begin{array}{c}\text { \# of Cases } \\
\boldsymbol{P}_{\boldsymbol{s}} \boldsymbol{>} \mathbf{4 0 \%}\end{array}$ & $\begin{array}{c}\text { \# of Cases } \\
\boldsymbol{P}_{\boldsymbol{s}} \leq \mathbf{4 0 \%}\end{array}$ \\
\hline Normal & 29 & 9 \\
\hline Hypokinetic & 4 & 3 \\
\hline Akinetic & 2 & 13 \\
\hline
\end{tabular}

In order to further evaluate our technique's performance, the Receiver Operating Characteristic (ROC) curve has also been plotted. The area under the ROC curve, which describes the accuracy of the test (i.e., its ability to correctly classify normal and abnormal cases), is 0.784 .

In addition, the histogram of the peak phase $\phi_{\max }^{s}$, as calculated for each segment of each cine-loop, is displayed in Fig. 5.

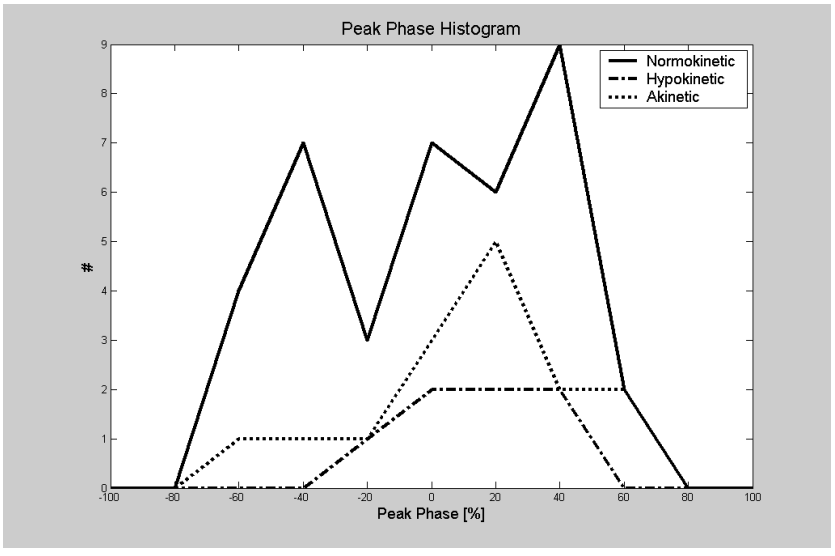

Figure 5. The histogram of the peak phase $\phi_{\max }^{s}$ over all the segments diagnosed as normal (solid line), hypokinetic (dash-dotted line) and akinetic (dotted line). While the values for normal tissue are symmetrically distributed around phase $0 \%$, the peak phase for hypokinetic and akinetic tissue is noticeably biased towards positive values.

\section{Discussion and conclusions}

As to the procedure's validity - the huge difference between the MSE calculated for $R(m, n)$ and $D(m, n)$ supports our assertion, that $R(m, n)$ is the dominant factor determining the attenuation. This phenomenon can also be seen in the examples in Fig. 2 and Fig. 3.

As to the perfusion estimation - the results in Table 1, as well as the relatively large area under the ROC curve (an area of 1.0 represents a perfect test, whereas an area of 0.5 represents a worthless test, so that our test is pretty good), show great promise for our method. It should be stressed, that even theoretically, the correlation between local perfusion and local contractility is not perfect (due to hibernation, stunned myocardium, and so forth).

Moreover, when reviewing the attenuation corrected IBS, one can clearly see cyclic variations, whose cycle length corresponds to the heartbeat. As shown in Fig. 5, the peak phase of both hypokinetic and akinetic tissue tends to be positive, whereas there is no such tendency in normal tissue. This effect, which requires further research, might provide us with new insight into the physiology of blood supply to the myocardium.

\section{Acknowledgements}

This work has been partially funded by a "Nophar" Grant, of the Israeli Ministry of Commerce and Industry, and by the Kodesh Fund of Tel Aviv University.

\section{References}

[1] Desco M, Ladesma-Carbayo MJ, Santos A et al. Myocardial perfusion assessment with contrast echocardiography. SPIE Proc 2001; 4325:514-522.

[2] Lafitte S, Higashiyama A, Masugata $\mathrm{H}$ et al. Contrast echocardiography can assess risk area and infarct size during coronary occlusion and reperfusion: Experimental validation. J Am Coll Cardiol 2002; 39:1546-1554.

[3] Mor-Avi V, Akselrod S, David D, Keselbrener L. Digital path-dependent recompensation of contrast-enhanced echocardiographic images. Ultrasound Med Biol 1992; 18:831-842.

[4] Mor-Avi V, Akselrod S, David D, Keselbrener L, Bitton Y. Myocardial transit time of the echocardiographic contrast media. Ultrasound Med Biol 1993; 19:635-648.

[5] Zwirn G, Beeri R, Gilon D et al. Quantitative evaluation of left ventricular local perfusion in contrast echocardiography. Ultrasound Med Biol 2005 (submitted).

[6] Zwirn G, Akselrod S. Adaptive brightness transfer functions in echocardiography. Ultrasound Med Biol 2005; 31:649-661.

[7] Zwirn G, Beeri R, Gilon D et al. Novel procedure for attenuation correction in contrast echocardiography. Ultrasound Med Biol 2005 (submitted).

Address for correspondence

Gil Zwirn

The Abramson Center of Medical Physics, Tel Aviv University Tel Aviv 69978, Israel 